\title{
Melody to musical notation translating system
}

\begin{abstract}
This paper presents a system that translates the captured or recorded melody into musical notations automatically and instantly into a developed stave whereby a musician can compose music directly without any extra process or procedure, in real-time environments. In this translating system, the frequency of a captured or recorded melody is first analyzed through a microphone or musical instrument for its fundamental frequency. The analyzed fundamental frequency is then compared with the predefined frequency of musical notes. The matched musical note frequency will be distinguished at the developed musical stave interface instantly. This developed system can facilitate a composer in automatically translate his melody to musical notes without having to manually writing it down based on the melody he plays.
\end{abstract}

Keyword: Fundamental frequency; Melody; Musical composing system; Musical notation; Musical translating system 